\title{
LA INTERPRETACIÓN JUDICIAL EN ESPAÑA EN UN MOMENTO DE CAMBIO
}

\author{
María Jesús Blasco Mayor \\ blascom@uji.es \\ Universitat Jaume I \\ Maribel del Pozo Triviño \\ mdelpozo@uvigo.es \\ Universidade de Vigo
}

\section{Resumen}

La publicación en la Unión Europea de la Directiva 2010/64/UE del Parlamento Europeo y del Consejo, de 20 de octubre, relativa al derecho a interpretación y traducción en los procesos penales ha marcado un antes y un después en una gran cantidad de aspectos relacionados con la interpretación en sede judicial y policial. Esta norma tiene como principal objetivo garantizar la interpretación judicial de calidad durante todo el proceso, como parte del derecho a la defensa y a un juicio justo.

España, como Estado Miembro de la UE, tiene la obligación de transponer la norma europea a su derecho interno. Se trata, pues, de un momento histórico en el que confluyen dos factores principales: la necesidad de cambiar la legislación para adaptarla a la nueva norma y la necesidad de implementar medidas para garantizar el cumplimiento de los nuevos mandatos.

En el presente artículo se realiza una revisión del estado de la cuestión sobre la interpretación judicial en España desde el punto de vista de la legislación y de la provisión de servicios, y se analizan las medidas que debe tomar nuestro país para garantizar que la interpretación en los tribunales de justicia se lleva a cabo con las debidas garantías. Estas medidas incluyen la formación de intérpretes y de operadores judiciales, la creación de sistemas de acreditación y registros, así como la consolidación del perfil profesional de los intérpretes. 


\begin{abstract}
"Legal Interpreting in Spain at a Turning Point"

The publication in the European Union of Directive 2010/64/EU of the European Parliament and of the Council of 20 October 2010 on the right to interpretation and translation in criminal proceedings has been a turning point in a great number of aspects related to court and police interpretation. The main objective of the Directive is to ensure quality legal interpretation throughout the process, as part of the right to defense and to a fair trial.

Spain, as a Member State of the EU, has the obligation to transpose this European Directive into its domestic law. Therefore, this is a historic moment in which two main factors converge: the need to change the legislation to bring it in line with the new Directive and the need to implement measures to ensure compliance with new mandates.

This paper reviews the present state of legal interpretation in Spain from the point of view of legislation and that of service provision and analyses the measures that Spain should take to ensure that court and police interpretations are carried out with due guarantees. These measures include the training of interpreters and legal operators, the creation of accreditation systems and records, as well as the consolidation of the professional profile of interpreters.
\end{abstract}

Palabras clave: Interpretación judicial. Profesionalización. Formación. Acreditación. Registro.

Keywords: Legal interpreting. Professionalization. Training. Accreditation. Register.

Editorial article, received on December 1, 2014. 


\section{Introducción}

Uno de los pilares sobre los que se asienta el marco internacional y europeo de derechos humanos es la prohibición de discriminación, tanto directa como indirecta. En este sentido, la provisión de intérpretes a personas inmersas en procesos judiciales que desconocen el idioma o los idiomas oficiales es una medida clave para asegurar su no discriminación en el acceso a la justicia.

Como parte de su compromiso con la creación de un espacio de libertad, seguridad y justicia, la UE ha trazado un plan de trabajo y ha tomado una serie de medidas que se describen en el considerando 9 de la Directiva 2013/48:

El 30 de noviembre de 2009, el Consejo adoptó una Resolución relativa al plan de trabajo para reforzar los derechos procesales de sospechosos y acusados en los procesos penales (en lo sucesivo, "plan de trabajo"). Mediante un enfoque gradual, dicho plan reclama la adopción de medidas relativas al derecho a la traducción e interpretación (medida A), el derecho a ser informado de sus derechos y de la acusación (medida B), el derecho a asistencia letrada y asistencia jurídica gratuita (medida C) y el derecho a la comunicación con familiares, empleadores y autoridades consulares (medida D), así como a salvaguardias especiales para aquellos sospechosos o acusados que sean personas vulnerables (medida E). En el plan de trabajo se subraya que el orden en el que se mencionan los derechos es solamente indicativo, por lo que puede modificarse en función de las prioridades. El plan de trabajo está concebido para funcionar como un conjunto; solo cuando se ejecutan todos sus componentes se alcanzan plenamente sus beneficios.

La medida A se ha visto materializada con la publicación de la Directiva 2010/64/UE del Parlamento Europeo y del Consejo, de 20 de octubre, relativa al derecho a interpretación y traducción en los procesos penales (en lo sucesivo, "la Directiva") que establece unas normas mínimas comunes para los países de la Unión Europea sobre el derecho a interpretación y a traducción en los procesos penales y obliga a los Estados Miembros a transponerla antes de octubre de 2013. En primer lugar, la Directiva establece que deberá facilitarse el derecho a interpretación y traducción a toda persona que no hable o no entienda la lengua del proceso; dicho derecho se aplicará a partir del momento en que se comunique a las personas que son sospechosas o que están acusadas de haber cometido una infracción penal y hasta la finalización del proceso. 
Asimismo, la nueva norma europea establece que deberá facilitarse un intérprete a las personas inmersas en un proceso penal que tengan que comunicarse con su abogado en relación directa con cualquier interrogatorio o vista judicial durante un proceso. Otro requisito que establece la Directiva es que los Estados Miembros también deberán facilitar a los sospechosos o acusados la traducción de todos los documentos que resulten esenciales, entre los que se incluyen "cualquier resolución que prive a una persona de libertad, escrito de acusación y sentencia". ${ }^{1}$

La finalidad de la Directiva es que los Estados Miembros pongan en marcha mecanismos que garanticen la calidad de la interpretación y de la traducción en los procesos penales a fin de que se pueda garantizar el derecho de defensa y el derecho a un juicio justo, para reforzar la confianza mutua entre sí.

Como mecanismo para garantizar dicha calidad, la Directiva insta a los Estados a crear "uno o varios registros de traductores e intérpretes independientes debidamente cualificados". Con el fin de cumplir con la recomendación de la Directiva, los Estados Miembros deberán crear registros cuyos miembros cumplan una serie de requisitos que garanticen su solvencia profesional, por lo que, a nuestro entender, deberán contar con la adecuada formación y será necesario, además, que pasen unas pruebas objetivas de acreditación. La noción de "independiente" que cita la Directiva no está totalmente clara ya que puede aludir tanto a que sean personas no vinculadas a ningún organismo, como empresas o instituciones, como a que no tengan conflicto de intereses con la persona o personas para las que interpretan.

España, como Estado Miembro de la UE, también debe trasponer la Directiva a su legislación nacional, ya que en la actualidad cuenta con una legislación absolutamente desfasada, la cual da pie a la existencia de unos sistemas de provisión de servicios de traductores e intérpretes que en modo alguno garantizan la tutela judicial efectiva y el derecho de defensa, que son obligaciones del Estado de Derecho (véase apartado 2).

Como parte de ese plan de trabajo y de esas medidas que la UE ha establecido y que se describen más arriba, las garantías que establece la Directiva 2010/64/UE han sido reforzadas además por otras 3 directivas:

1. Para un estudio detallado de la Directiva 2010/64/UE, véase el artículo de Hertog en este volumen. 
- Directiva 2012/13/UE del Parlamento Europeo y del Consejo de 22 de mayo de 2012 relativa al derecho a la información en los procesos penales.

- Directiva 2012/29/UE del Parlamento Europeo y del Consejo de 25 de octubre de 2012 por la que se establecen normas mínimas sobre los derechos, el apoyo y la protección de las víctimas de delitos, y por la que se sustituye la Decisión marco 2001/220/JAI del Consejo.

- Directiva 2013/48/UE del Parlamento Europeo y del Consejo de 22 de octubre de 2013 sobre el derecho a la asistencia de letrado en los procesos penales y en los procedimientos relativos a la orden de detención europea, y sobre el derecho a que se informe a un tercero en el momento de la privación de libertad y a comunicarse con terceros y con autoridades consulares durante la privación de libertad.

Todas ellas consideran el derecho a interpretación y traducción una garantía de facto para que víctimas y encausados puedan hacer efectivos sus derechos.

En el presente artículo se realiza una somera revisión de la situación actual de la interpretación judicial y policial en España, ${ }^{2}$ desde el punto de vista legislativo y de provisión de servicios (RITAP 2011, Ortega Herráez 2011, Del Pozo Triviño 2013, Blasco Mayor 2013, Del Pozo Triviño \& Borja Albi 2014) y se analizan diversos mecanismos para la creación de registros de intérpretes y traductores judiciales con el fin de aportar información sobre los pasos que debería seguir nuestro país para transponer correctamente las normas europeas antes mencionadas (Corsellis, Cambridge, Glegg \& Robson 2007; Blasco Mayor 2013; Del Pozo Triviño 2013; Del Pozo Triviño \& Borja Albi 2014). Asimismo, se analizan diversos modelos de formación y acreditación en interpretación judicial (Blasco Mayor, Del Pozo Triviño, Giambruno, Martin, Ortega Arjonilla, Rodríguez Ortega \& Valero Garcés 2013; Giambruno 2014; Blasco Mayor 2013; Mikkelson 2014) y de formación de operadores judiciales para trabajar con intérpretes (Blasco Mayor 2014; Corsellis, Clement y Vanden Bosch 2011). En todos los puntos tratados se dedica especial atención tanto a las particularidades de las lenguas que hemos llamado "de menor difusión" y que son, en la actualidad, las más presentes en nuestros juzgados, como a la regulación y consolidación profesional de los intérpretes (Del Pozo Triviño 2013, Blasco Mayor 2013, Mikkelson 1996).

2. En adelante, todas las referencias que se hacen en el artículo a la interpretación judicial incluyen la interpretación policial. 


\section{Situación actual de la interpretación y traducción en procesos penales en España}

\subsection{Legislación española sobre interpretación y traducción en procesos penales}

En el presente apartado se hace una breve revisión de la legislación española sobre el derecho a interpretación y traducción, especialmente en la jurisdicción penal. La Ley de Enjuiciamiento Criminal (LECr) al referirse a la fase de instrucción preliminar, llamada "sumario" dentro del procedimiento ordinario, que se sigue para los delitos castigados con más de nueve años de prisión, dice literalmente en su artículo 440:

Si el testigo no entendiere o no hablare el idioma español, se nombrará un intérprete, que prestará a su presencia juramento de conducirse bien y fielmente en el desempeño de su cargo. Por este medio se harán al testigo las preguntas y se recibirán sus contestaciones, que éste podrá dictar por su conducto. En este caso, la declaración deberá consignarse en el proceso en el idioma empleado por el testigo y traducido a continuación al español.

Más adelante, refiriéndose a la cualificación y acreditación de los intérpretes, el artículo 441 dice así:

El intérprete será elegido entre los que tengan títulos de tales, si los hubiere en el pueblo. En su defecto, será nombrado un maestro del correspondiente idioma, y si tampoco le hubiere, cualquier persona que lo sepa.

Vemos, por lo tanto, que la LECr, promulgada en 1882, en teoría establece un orden de prelación, ya que dice que el juez elegirá primero "a los que tengan el título", después "al maestro del correspondiente idioma" y, por último, "a cualquier persona que lo sepa". Sin embargo, el procedimiento abreviado, el cual se aplica cuando las penas privativas de libertad son inferiores a nueve años, ni siquiera menciona el orden de prelación que se establecía para el procedimiento ordinario, sino que dice literalmente que el intérprete no necesitará título oficial:

Cuando los imputados o testigos no hablaren o no entendieren el idioma español, se procederá de conformidad con lo dispuesto en los artículos 398, 440 y 441 , sin que sea preciso que el intérprete designado tenga título oficial.

Tal y como señala el Libro blanco de la traducción y la interpretación institucional (RITAP 2011: 19), "Los artículos de la LECr se han quedado obsoletos, son propios del siglo XIX y no reflejan la transformación que ha sufrido la sociedad española”. Por otra parte, la Ley Orgánica del Poder Judicial, en su artículo 231, establece que los jueces y magistrados tienen potestad para nombrar intérprete a cualquier persona durante las actuaciones orales. 
Como ya se ha mencionado, España debe adaptar esta legislación a las nuevas normas europeas. Sin embargo, en la fecha de publicación de este artículo, las directivas mencionadas en el apartado de Introducción aún no se han transpuesto a la legislación nacional española, de modo que el derecho manifiesto que recogen las normas europeas todavía no se garantiza en el contexto español. ${ }^{3}$

\subsection{Provisión de servicios de interpretación en los procesos penales}

$\mathrm{Al}$ amparo de este marco legal actual tan desfasado y permisivo, que ofrece a los jueces y magistrados la posibilidad de nombrar traductor/intérprete judicial "a cualquier persona que sepa la lengua" del proceso, sin necesidad de que se acredite ningún tipo de formación ni de capacitación profesional, no es de extrañar que surjan modelos de provisión de servicios que no garantizan la calidad de las traducciones y/o interpretaciones. En los párrafos que siguen se exponen los principales modelos de contratación de traductores e intérpretes que existen en España en la actualidad (RITAP 2011: 47-71, Ortega Herráez 2011). Según Ortega Herráez (2011: 95), existen en nuestro país tres sistemas de contratación de traductores e intérpretes en el ámbito judicial. En líneas generales, y sin tener en cuenta las peculiaridades de las Comunidades Autónomas que tienen transferidas las competencias de justicia, y que son la mayoría, los tres modelos de provisión de servicios de interpretación en España son actualmente los siguientes:

- Modelo tradicional: en este modelo conviven los intérpretes en plantilla (que acceden al puesto mediante un concurso-oposición) con intérpretes freelance (a los que se recurre cuando la carga de trabajo es muy elevada y cuando se requiere interpretación en idiomas para los que no hay intérpretes en plantilla).

- Subcontratación de servicios ("modelo de contratas"): las administraciones publican una licitación a la que se presentan empresas privadas. El hecho de que la empresa actúe de intermediaria hace que se reduzcan considerablemente las tarifas que reciben los intérpretes, lo cual a su vez hace que muchos profesionales no acepten las condiciones impuestas

3. Al cierre de este artículo, el proceso de trasposición de las directivas se estaba desarrollando, principalmente a través de dos proyectos de ley: estatuto de la víctima, que incorpora por primera vez en España el derecho de las víctimas a intérprete, y la modificación de la LECr, que reconoce el derecho de las víctimas y encausados a intérpretes profesionales y cualificados. 
por las empresas concesionarias y que estas contraten a personas sin apenas formación ni experiencia. ${ }^{4}$

- Gestión integral pública de servicios de traducción e interpretación judicial: en este modelo, exclusivo de la provincia de Las Palmas, un único traductor-intérprete en plantilla se encarga de coordinar a todos los demás intérpretes. Este modelo, a pesar de no ser perfecto, tiene ciertas ventajas, como el hecho de que, al no haber intermediarios, los traductores e intérpretes cobren las tarifas íntegras que paga el Estado y de que hay un intérprete que ejerce cierto control sobre la calidad.

Desafortunadamente, el modelo que se ha impuesto en los últimos años en la mayor parte del territorio español es el llamado "modelo de contratas", mediante el cual la Administración, a través de licitación pública, contrata a empresas privadas los servicios de traducción e interpretación en los tribunales y también en la policía (Ortega Herráez \& Foulquié Rubio 2008: 125). Dichas empresas son las encargadas de escoger a los traductores e intérpretes, de establecer los requisitos para su contratación (mínimos en la mayoría de los casos), así como de determinar sus condiciones de trabajo: horario, remuneración, y demás. Este modelo de contratas está redundando de forma muy negativa en la calidad de las interpretaciones así como en la percepción social que se tiene de la profesión. Por todo ello, existen numerosos colectivos que, tanto desde el mundo profesional como académico, están luchando por poner freno a esta práctica al tiempo que proponen fórmulas alternativas que velen por la calidad de la traducción y la interpretación, y por la racionalización del gasto (De Luna Jiménez de Parga 2009).

Una vez analizada la situación en la que se encuentra actualmente la interpretación en los tribunales españoles con respecto a la legislación y a la provisión de servicios, podemos concluir que España debe recorrer un largo camino para poder cumplir con los preceptos de la Directiva 2010/64/UE.

\section{Registros de intérpretes judiciales}

Como ya se ha mencionado, uno de los mecanismos que la Directiva propone a los Estados Miembros para asegurar la calidad de la interpretación judicial es la creación de "uno o varios registros de traductores e intérpretes independientes debidamente cualificados" (Art.5.2). Así pues, los Estados Miembros deberán crear registros cuyos miembros cumplan una serie de requisitos que garanticen su solvencia profesional. En los siguientes subapartados se abordan

4. Existen numerosas quejas y denuncias sobre los perjuicios que causa la contratación de traductores e intérpretes no profesionales en diversos ámbitos de la justicia (Handi 2012; De Luna Jiménez de Parga 2009). 
algunas cuestiones importantes relacionadas con los registros de traductores e intérpretes y se realiza una propuesta de registro para España.

\subsection{Definición del concepto}

Según Corsellis, Cambridge, Glegg \& Robson (2007: 140), una profesión es un grupo de personas que comparten un saber hacer experto, que profesan un código de valores para proteger a sus clientes, sus conocimientos y colegas y van más allá del interés personal de sus miembros. Para cumplir los requisitos establecidos en dicho código, los profesionales establecen sistemas nacionales, transparentes, sistemáticos, que rinden cuentas, entre los que se incluyen la selección, formación, acreditación, pertenencia a un registro, promoción de buenas prácticas, control de la calidad y establecimiento de procedimientos disciplinarios. Como indican las autoras, esta definición ya aparecía en el primer proyecto europeo sobre el derecho procesal a interpretación en la justicia europea, Grotius 98/GR/131 (Hertog 2001), sobre la equivalencia de estándares de interpretación y traducción en todos los Estados Miembros, y fue aceptada por la Comisión Europea (Corsellis et al 2007: 140). Las autoras explican que no se refieren a un mero listado como registro:

[...] such a register is therefore, not a list or a directory but the public manifestation of a professional structure and of its integrity (op.cit.:141).

Más recientemente, el equipo de expertos europeos del proyecto Qualitas ${ }^{5}$ (Giambruno 2014: 250) define el concepto de registro profesional como sigue:

An independent voluntary or statutory body that registers and makes available the details of individuals who meet its criteria in terms of qualifications, experience and security clearance, and have agreed to observe its code of ethics/conduct along with its disciplinary procedures when any breach of the code is alleged. A professional register goes further than just a database or list.

Este concepto de registro como órgano "oficial" de profesionales cualificados e independientes, que se rige por normas oficiales a la vez que garantiza la independencia de los profesionales registrados, comprobando cualificaciones, experiencia, antecedentes penales y el cumplimiento de un código ético, y no un mero listado o base de datos, es el que inspira la Directiva 2010/64/UE. En el ámbito europeo, la creación de registros nacionales tiene como propósito establecer un sistema que permita a las autoridades de los diferentes Estados Miembros identificar y localizar intérpretes cualificados e independientes en

5. http://www.qualitas-project.eu/ 
todos los países de la UE sin tener que dudar de la calidad o legitimidad de sus servicios. Para lograr este objetivo, tiene que haber cierta uniformidad o armonización en cuanto a los criterios mínimos que deben reunir los profesionales de la interpretación en cada uno de los Estados Miembros. Un registro que no garantice la calidad necesaria no contribuye a la confianza mutua ni a la seguridad jurídica deseadas (Blasco Mayor et al. 2013, Blasco Mayor 2013).

A continuación analizaremos la situación actual en España y las distintas posibilidades sobre la creación y gestión de un registro de intérpretes y traductores judiciales.

\subsection{Situación actual en España}

En la actualidad existe en España un listado de traductores-intérpretes jurados gestionado por el Ministerio de Asuntos Exteriores y de Cooperación. Para constar en el listado es necesario haber superado las pruebas convocadas por la Oficina de Interpretación de Lenguas (OIL), o haber obtenido el reconocimiento de dicha Oficina por haber cursado y superado determinados créditos en traducción jurídica e interpretación de la extinta Licenciatura en Traducción e Interpretación ${ }^{6}$ (Orden AEX/1971/2002, de 12 de julio, por la que se establecen los requisitos y el procedimiento para la obtención del nombramiento de intérprete jurado por los licenciados en Traducción e Interpretación). En cuanto a las pruebas realizadas por la OIL para conceder el título de traductor-intérprete jurado (Vigier Moreno 2010: 26), se trata de una traducción directa general, una traducción inversa de un texto jurídico y una entrevista con los candidatos para asegurarse de que son capaces de hablar las lenguas de trabajo con fluidez. Ninguna de estas pruebas está relacionada con conocimientos sobre el ámbito judicial ni con la capacidad de interpretar, por lo que parece claro que la palabra "intérprete" no debería formar parte del título ya que no se comprueba la capacidad de los candidatos para interpretar, y solo conduce a mayor confusión con respecto al perfil profesional de los intérpretes en España. ${ }^{7}$

6. "Licenciatura" era la denominación del título que ha pasado a denominarse "grado" tras las modificaciones introducidas con la adaptación al Espacio Europeo de Educación Superior.

7. Originalmente el título se denominaba "intérprete jurado", sin la palabra traductor, de introducción reciente. Se ha mantenido la denominación "intérprete" como un vestigio del origen del título, otorgado a los primeros intérpretes de las Américas y que data del siglo XVI (Peñarroja Fa 2004). Dicha denominación resulta anacrónica y no se adecúa a la realidad profesional europea (Blasco Mayor 2013). 
La OIL publica en su web un listado de traductores-intérpretes jurados que puede ser consultado por cualquier ciudadano que requiera de los servicios de estos profesionales. Está ordenado por lenguas y se limita a proporcionar el nombre y los datos de contacto de las personas que ostentan el título de traductor-intérprete jurado. Muy pocos titulados trabajan como intérpretes en el ámbito de la justicia penal; la mayoría trabajan en casos civiles y en traducciones juradas de documentos de diversa índole por tratarse de trabajos bien remunerados y con prestigio social.

La OIL no realiza ningún control de calidad sobre el desempeño profesional de las personas a quienes acredita ni se encarga de la formación continua y promoción profesional de sus titulados. Las características de las pruebas o el panorama profesional en el que habitualmente se circunscribe la actividad de los traductores-intérpretes jurados, totalmente al margen de los distintos perfiles que se dibujan en el ámbito judicial, no pueden garantizar apriorísticamente, o por sí mismas, que sus titulados estén capacitados para actuar como intérpretes judiciales.

En España, pues, no existe registro ni listado alguno de intérpretes judiciales. Puesto que, como se ha indicado en el apartado 2.2, la provisión del servicio se ha licitado a empresas privadas, son estas quienes deciden a quién enviar a trabajar como intérprete. En la mayoría de los casos, las personas que actúan como intérpretes no son profesionales ni traductores-intérpretes jurados, no han cursado estudios de Traducción e Interpretación, ni estudios superiores. Este modelo de gestión externa del servicio ha conducido al desgarro del tejido profesional en el ámbito judicial, que puede mejorar sensiblemente si se desarrolla un nuevo modelo que siga los mandatos de la Directiva 2010/64/UE, comenzando por el diseño y creación de un registro profesional de intérpretes y traductores judiciales en España según los mecanismos ya aprobados por la Comisión Europea en 2001.

\subsection{Propuesta de registro para España}

Para nuestra propuesta de un registro profesional en España nos basaremos en el modelo ya propuesto en el informe elaborado por la Conferencia de Centros y Departamentos Universitarios de Traducción e Interpretación de España (en adelante CCDUTI) para el Ministerio de Justicia español sobre la transposición de la Directiva 2010/64/UE (Blasco Mayor et al. 2013), en el que participaron activamente las autoras del presente artículo. Dicho informe toma como referencia las normas establecidas en el National Register of Public Service Interpreters (NRPSI) del Reino Unido, por tratarse del registro 
de referencia en toda Europa, y con toda probabilidad el modelo de registro en el que se inspira la Directiva 2010/64/UE, adaptándolas a la realidad española:

a) El registro debe tener normas claras y transparentes.

b) Sus miembros deben ser intérpretes y/o traductores cualificados e independientes.

c) Deben someterse a un código deontológico.

d) Deben estar libres de antecedentes penales.

e) Deben abonar unas tarifas a modo de colegio profesional.

f) Deben renovar periódicamente la pertenencia al mismo. Se deben establecer pautas para la renovación, entre las que pueden incluirse la experiencia demostrable en el ámbito judicial y la realización de cursos de formación continua, o bien volver a examinarse si esos criterios de renovación no se han cumplido.

g) El uso del registro debe ser gratuito.

h) Se podrán contemplar distintas categorías según las lenguas y el tipo de acreditación del candidato.

i) El registro debe ser nacional, si bien debido a que las comunidades autónomas con lenguas propias tienen transferidas las competencias de Justicia, podría pensarse también en registros que incluyeran a profesionales que trabajen con dichas lenguas.

El acceso al registro debería realizarse en todos los casos mediante prueba de acreditación fiable y validada, tal y como se explica en el apartado 5. Puesto que el Ministerio de Justicia es la institución responsable de la transposición de la Directiva, y por tanto de su cumplimiento, amén de la provisión del servicio, parece lógico pensar que debería ser también responsable del proceso de acreditación. Para su diseño y el proceso de evaluación, el Ministerio debe contar con la colaboración de expertos académicos y profesionales. Alternativamente, la acreditación podría ser administrada por un instituto interuniversitario creado a tal efecto, mediante convenio con el Ministerio de Justicia.

Otra posibilidad, considerando la organización territorial, política y administrativa del estado español en comunidades autónomas, la mayoría de ellas ${ }^{8}$ con competencias en la administración de justicia, es la creación de colegios profesionales de traductores e intérpretes en cada comunidad autónoma, encargados de la provisión del servicio y la gestión del registro. Esta modalidad ya se utiliza para la gestión y la provisión del servicio de los abogados

8. Andalucía, Aragón, Asturias, Canarias, Cantabria, Cataluña, Comunidad Valenciana, Galicia, Madrid, Navarra, País Vasco, La Rioja. Comunidades no transferidas: Castilla y León, Castilla-La Mancha, Extremadura, Murcia, Ceuta y Melilla. Fuente: https:// www.administraciondejusticia.gob.es/paj/PA_WebApp_SGNTJ_NPAJ/descarga/08c_ Doc_Estad\%C3\%ADstico_Traspasos_Competencias_Admón_de_Justicia. pdf?idFile=f96d9863-3b11-49a8-a64f-330eeac35158 (consultada el 5/11/2014). 
del turno de oficio en la administración de justicia española. El papel de los colegios profesionales en España tiene una larga tradición en el desarrollo y la evolución de las profesiones liberales. Según la legislación española vigente sobre colegios profesionales, que data del año 1974 (Ley 2/1974, de 13 de febrero, sobre Colegios Profesionales), ${ }^{9}$ en su art. 1.1, "los colegios profesionales son Corporaciones de derecho público, amparadas por la ley y reconocidas por el Estado, con personalidad jurídica propia y plena capacidad para el cumplimiento de sus fines", y según 1.3:

...son fines esenciales en dichas Corporaciones la ordenación del ejercicio de las profesiones, la representación institucional exclusiva de las mismas cuando estén sujetas a colegiación obligatoria, la defensa de los intereses profesionales de los colegiados, y la protección de los intereses de los consumidores y usuarios de los servicios de sus colegiados, todo ello sin perjuicio de la competencia de la Administración Pública por razón de la relación funcionarial.

Por la estructura y naturaleza jurídica de los propios colegios, serían estos, de hecho, las instituciones mejor equipadas para asumir las competencias que se derivan de la Directiva. Por un lado, en su seno podría acogerse el registro, y al igual que en el caso de los abogados del turno de oficio, gestionar el servicio abasteciendo de intérpretes y traductores registrados para los juzgados y policía de la zona. Por otro lado, en colaboración con expertos de las universidades, y a través de un Consejo General de Colegios de Traductores e Intérpretes, podrían asimismo encargarse de los procesos de comprobación de antecedentes penales de los candidatos, administración y evaluación de los exámenes específicos relativos a la acreditación requerida para acceder al registro de intérpretes y traductores judiciales a la que se alude en el apartado 5. Además, los colegios se encargarían de velar por la calidad de los servicios que prestan sus colegiados, puesto que es una de sus misiones principales, cooperando con las autoridades judiciales en el establecimiento de mecanismos de control de la calidad, tanto apriorísticos (acceso mediante examen, formación continua de sus miembros) como a posteriori [evaluación de grabaciones y otros mecanismos de control de la calidad (Vidal Fernández 2007, Arangüena Fanego 2007), aplicación de procedimientos disciplinarios, etc.], en consonancia con el artículo 2, apartado 8, el artículo 3, apartado 9; y el artículo 5 de la Directiva. Por último, y en cooperación con los colegios de abogados, escuelas judiciales y academias de policía, se podrían impartir

9. Última actualización, publicada el 23/12/2009, en vigor a partir del 27/12/2009. 
cursos formativos a estos operadores judiciales, tal y como establece la Directiva en su artículo 6 (véase apartado 6 del presente artículo).

Como quiera que la creación de colegios en las comunidades autónomas y el desarrollo de un examen requieren, además del desarrollo de normativa legal, de un tiempo considerable de ejecución, se podría crear un primer registro "provisional" y establecer una etapa de transitoriedad que deberá tener una fecha de caducidad, a cuyo vencimiento las personas interesadas en seguir en el registro deberán haber cumplido una serie de requisitos. Durante dicha etapa de transitoriedad se podría contemplar en principio la incorporación al registro "provisional" en los siguientes casos:

- Graduados/Licenciados en Traducción e Interpretación que puedan demostrar experiencia profesional real en el ámbito judicial.

- Traductores-intérpretes jurados (MAEC) que puedan demostrar experiencia real en el ámbito judicial.

- En el caso de las lenguas de menor difusión, se podría incluir a personas con experiencia demostrada o formación específica adquirida en otros países.

Durante dicha etapa de transitoriedad, y de manera excepcional, se podría permitir el acceso al registro a otros titulados universitarios que tengan experiencia acreditada en el campo con el fin de no bloquear el acceso de los profesionales que ya trabajan y lo hacen con solvencia.

Para aquellas lenguas en las que no existen Grados/Licenciaturas en Traducción e Interpretación, ni filologías, deberá exigirse a las personas que deseen formar parte del registro que realicen un curso de especialización, que pueden realizar las universidades en colaboración con las asociaciones profesionales. ${ }^{10}$ En dichos casos, el Gobierno deberá incentivar y apoyar económicamente a las personas que hablan las lenguas con mayor demanda para que cursen estos estudios.

Si se plantea un reconocimiento de traductores e intérpretes judiciales pertenecientes a registros de otros Estados Miembros de la Unión Europea, tendría que garantizarse la reciprocidad, es decir, que traductores e intérpretes judiciales españoles acreditados para el presente registro también sean reconocidos en esos mismos países.

Una forma de garantizar la incorporación de intérpretes cualificados en el sistema judicial, bien sea a través de colegios, o de registros, es el establecimiento en la ley de un arancel profesional, al igual que en Alemania, Austria y

10. Véase "cursos propios" en el punto 4.2 sobre Formación. Dichos cursos tienen como objetivo la formación de perfiles profesionales con demanda en la sociedad que carecen de docencia reglada en los títulos oficiales. 
otros países europeos, donde la tarifa que se abone a los intérpretes por hora/ jornada está establecida en la ley o en el instrumento jurídico que regule la implementación de la Directiva. Con esta medida se evitarán los abusos que actualmente cometen las empresas adjudicatarias de las licitaciones públicas y se garantizará que los profesionales reciban una contraprestación justa por un trabajo que requiere gran especialización, fomentando así la consolidación de la profesión, la atracción de talento y la calidad, que redundarán en todo el proceso judicial. Solo así se podrán garantizar los derechos procesales, y cumplir los mandatos establecidos en la Directiva 2010/64/UE.

En España existen varios antecedentes. Concretamente de aplicación en todos los órdenes del ámbito judicial, existen los aranceles de los procuradores de los tribunales, contemplados en el Real Decreto 1373/2003, de 7 de noviembre, por el que se aprueba el arancel de derechos de los procuradores de los tribunales, por el cual se establecen tarifas muy detalladas según los ámbitos y el volumen del servicio de dichos profesionales.

En todo caso, el registro deberá observar las máximas de cualificación e independencia de sus miembros, por lo que no deberá ser dependiente ni estar al servicio particular de ningún organismo o entidad judicial o policial.

\section{Formación en interpretación judicial}

Han pasado ya algunos años desde que Gile escribiera:

[...] the training of professional translators and interpreters is still based essentially on professional experience, introspection, intuition and negotiations between trainers on methods and modalities rather than on research (2009:3).

En los últimos años se ha experimentado un crecimiento exponencial en investigación tanto sobre didáctica de la traducción y la interpretación general como sobre la didáctica de la traducción y la interpretación especializadas $y$, por ende, de la interpretación judicial. Al mismo tiempo, en los últimos años la oferta formativa universitaria en traducción e interpretación ha crecido enormemente, tanto en España como fuera de nuestro país. No obstante, la oferta de formación en traducción e interpretación judicial sigue siendo escasa y en la mayoría de los casos se limita a unos módulos específicos dentro de los programas generales de grado y posgrado. En el presente apartado se analiza la oferta formativa existente en la actualidad en Europa, y más específicamente en España, y se realiza una propuesta de enseñanza de la 
traducción e interpretación judicial ${ }^{11}$ que pueda dar respuesta a la creciente demanda existente en la actualidad y que se verá incrementada una vez que se articulen los mecanismos para la transposición de la Directiva y la creación de registros de intérpretes cualificados.

\subsection{Formación reglada}

En numerosos países europeos (Reino Unido, Alemania, Austria, Países Bajos, Bélgica, Italia, etc.) existen estudios universitarios de Grado y Máster en traducción e interpretación. ${ }^{12}$ En cuanto a la formación de Máster en traducción e interpretación judicial, la mayoría de los Estados Miembros ofrece algún tipo de enseñanzas pero, o bien están integradas dentro de denominaciones más amplias, como Máster en traducción e interpretación, o bien se incluyen en másteres más específicos como son los dedicados a la formación en interpretación para los servicios públicos, la cual incluye, además de la judicial, la interpretación policial, sanitaria y en el ámbito educativo y de los servicios sociales. Por ejemplo, en el Reino Unido hay másteres que incluyen formación en TeI (Traducción e Interpretación) en los servicios públicos (como por ejemplo el de la University of Surrey), pero ninguno especializado exclusivamente en interpretación judicial. En Países Bajos, el Stichting Instituut van Gerechtstolken \& -Vertalers (SIGV) ${ }^{13}$ ofrece formación especializada y una acreditación específica para traductores e intérpretes judiciales en 20 idiomas.

En nuestro país, sobre 20 universidades públicas y algunas privadas ofrecen estudios de Grado en TeI (Baxter 2014) y algunas de ellas ofrecen estudios de Máster que incluyen formación en interpretación judicial y traducción jurídica. La formación de Máster disponible actualmente en España es la siguiente:

- Máster Universitario en Comunicación Intercultural, Interpretación y Traducción en los Servicios Públicos (Universidad de Alcalá de Henares), miembro de la Red de Másteres Europeos EMT Network.

- Máster en Traducción Jurídica e Interpretación Judicial (Universitat Autònoma de Barcelona).

11. Propuesta basada en el Informe realizado por Blasco et al. (2013) para el Ministerio de Justicia.

12. El proyecto OPTIMALE, Optimising Translator Training, financiado por UE, ha realizado un mapa interactivo que ofrece información actualizada sobre formación en traducción e interpretación en la UE (http://www.translator-training.eu/).

13. http://www.sigv.nl/ 
- Máster Universitario en Traducción Jurídico-Financiera (Universidad Pontificia Comillas de Madrid ICADE-ICAI), miembro de la Red de Másteres Europeos EMT Network.

- Máster Universitario en Traducción Institucional (Universitat d'AlacantUniversitat Jaume I - Universitat de València).

Ninguno de estos títulos está específicamente orientado a la interpretación en el ámbito judicial (si bien el de la Universidad Autónoma de Barcelona es el que presenta un perfil más específico). Sin embargo, es posible que todos ellos puedan actualizar sus contenidos para que aumenten los créditos dedicados a estas disciplinas. Existen hasta otros nueve másteres en traducción especializada ofrecidos por otras universidades españolas; algunos de ellos incluyen módulos de interpretación judicial o traducción jurídica, pero de forma más residual.

En relación con las lenguas de menor difusión, cabe destacar que el mencionado Máster Universitario en Traducción e Interpretación en los Servicios Públicos, de la Universidad de Alcalá de Henares, ofrece formación hasta en 10 pares de lenguas, con especial atención a lenguas consideradas de menor difusión que son, en realidad, las de mayor demanda en instancias judiciales. Las combinaciones lingüísticas en las que se imparte formación junto con el español son: alemán, árabe, búlgaro, chino, francés, inglés, portugués, rumano, ruso y polaco.

\subsection{Formación no reglada: cursos propios universitarios}

Un ejemplo de formación no reglada en el ámbito europeo es el Reino Unido, donde se ofrecen cursos universitarios de preparación para el examen de acreditación oficial del CIoL, el llamado Diploma in Public Service Interpreting (DPSI); tal es el caso del Diploma in Legal Interpreting ${ }^{14}$ de la Universidad de Middlesex, con 45 créditos, y formación en 14 pares de lenguas.

En España, la Ley Orgánica 6/2001, de 21 de diciembre, de Universidades, en su art. 34.3, otorga a las universidades, en uso de su autonomía, la posibilidad de dispensar enseñanzas conducentes a la obtención de diplomas o títulos propios, así como enseñanzas de formación a lo largo de toda la vida. Estos títulos propios parecen ser una vía adecuada para cubrir la laguna existente en la formación de intérpretes judiciales, pues como señala el reglamento de 29 de mayo de 2013 que regula estos títulos de la Universidad de La Laguna,

[...] estas enseñanzas, cuyo interés radica en responder, de manera ágil y eficaz, a las demandas sociales de tipo cultural, científico, artístico o profesional,

14. http://www.mdx.ac.uk/courses/undergraduate/legal-interpreting 
complementan el conjunto de enseñanzas curriculares oficiales y forman parte, junto con estas últimas, de la oferta docente de cada Universidad, contribuyendo, en consecuencia, a dotarla de un perfil propio. La posibilidad de impartir todas estas enseñanzas no oficiales cubre una importante laguna en la oferta universitaria, de forma que permite a la Universidad responder al reto de la creciente competitividad del mercado laboral que exige una mayor cualificación a todos los trabajadores.

En la actualidad, existen en España dos cursos propios de postgrado que incluyen formación en traducción e interpretación judicial, pero no de forma exclusiva, sino dentro de un programa general de interpretación para los servicios públicos (hospitales, centros educativos, servicios sociales, entre otros). Dichos cursos son:

- Diploma de especialización en Traducción e Interpretación para los Servicios Comunitarios (Universidad de La Laguna). ${ }^{15}$

- Diplomatura de Postgrado en Interpretación en los Servicios Públicos de Cataluña (Universitat Autònoma de Barcelona). ${ }^{16}$

En ambos cursos, se presta especial atención a las lenguas de menor difusión. El primero ofrece formación en inglés, francés, alemán y ruso; y el segundo en árabe, chino, rumano, ruso, inglés y francés.

\subsection{Propuesta de formación en interpretación judicial}

La formación de intérpretes judiciales debe basarse en tres pilares fundamentales. Por un lado, debe incluir el aprendizaje de las técnicas, modalidades y estrategias de interpretación en sede judicial y policial. Por otro lado, debe incluir formación sobre derecho comparado en las combinaciones lingüísticas pertinentes ${ }^{17} \mathrm{y}$, por último, debe abordar la deontología de la profesión (Dueñas González, Vásquez \& Mikkelson 1991: 202). De cara al cumplimiento de unos requisitos comunes para todos los traductores e intérpretes de la UE, consideramos que los contenidos y el formato de esta formación deberían estar sancionados por la propia CE con la colaboración y el asesoramiento de

15. http://experto.webs.ull.es/

16. http://www.uab.es/servlet/Satellite/postgrado/diplomatura-de-postgrado-en-interpretacion-en-los-servicios-publicos-de-cataluna-arabe-chino-rumano-ruso-ingles-frances-/datos-basicos-1206597472083.html/param1-2996_es/param2-2009/

17. Los intérpretes que trabajan en países con sistemas judiciales inquisitoriales (España, Francia, Italia), requieren más formación sobre el sistema procesal penal y la terminología judicial que los que lo hacen en países con sistemas adversariales (Reino Unido, Estados Unidos) (Giambruno 2014). 
expertos académicos y profesionales y, por supuesto, en consonancia con los distintos organismos acreditadores que se creen en cada Estado Miembro. ${ }^{18}$

Dicha formación específica podrían impartirla las universidades mediante la organización de Cursos propios y Diplomas, siguiendo el modelo de los que ya existen, con la colaboración de profesionales con experiencia en el sector. ${ }^{19}$ Con el fin de abaratar los costes que implica la constitución de tribunales en muchos pares de lenguas, y de garantizar la homogeneidad del proceso, podría establecerse un tribunal evaluador único para todo el país.

En el caso de las lenguas de menor difusión, la formación no estaría tan centrada en las propias lenguas, ya que sería prácticamente imposible disponer de formadores en todas las combinaciones lingüísticas. El alumnado que desee acceder a la formación debería acreditar conocimientos de la lengua española y de la lengua con la que tiene intención de trabajar como intérprete judicial, y dicha acreditación de conocimientos lingüísticos deberá hacerse de conformidad con criterios homogéneos previstos en la normativa establecida para tal fin.

En todos los casos la formación estaría siempre encaminada al ejercicio práctico de la profesión, por lo que, además de incluir ejercicios que simulen situaciones reales, también sería conveniente que incluyera prácticas tuteladas siempre por profesionales, así como visitas a los juzgados y a dependencias policiales.

\section{Sistema de acreditación de intérpretes judiciales}

Para hablar con propiedad sobre un proceso de acreditación en España, es necesario, en primer lugar, acotar conceptos. Según la RAE, acreditación es un "documento que acredita la condición de una persona y su facultad para desempeñar determinada actividad o cargo", y certificación es "un documento en que se asegura la verdad de un hecho". Creemos que el término "acreditación" en español sería el más apropiado en el contexto que nos ocupa. Siguiendo dichas definiciones, en España no existe un sistema o proceso de acreditación en interpretación judicial, entendiendo por tal un procedimiento previamente establecido, en el cual los candidatos deben demostrar una destreza suficiente, es decir, que ofrezca garantías de calidad, al realizar una serie de pruebas diseñadas con métodos fiables y basadas en la realidad profesional.

18. Véase informe final de Grotius project I (2001/GRP/015), Aequitas - Access to Justice across Language and Culture in the EU.

19. Véase artículo de Hertog en este mismo volumen. 
Dicho procedimiento o sistema, para ofrecer garantías de calidad, debe ser diseñado y evaluado por expertos externos.

Para el ámbito estadounidense citaremos a Mikkelson (2013: 66), quien apunta que las organizaciones son acreditadas y los individuos certificados. Además, incluye las licencias como alternativa a la certificación aunque éstas se refieren normalmente a autorizaciones institucionales concedidas a individuos que han demostrado ciertas competencias para desarrollar una actividad por un periodo de tiempo determinado. En las profesiones en las que existen licencias, estas son un requisito legal para ejercerlas, de modo que quien no tiene licencia no puede usar el título ni proveer el servicio. Por el contrario, una certificación suele ser un proceso voluntario al cual se somete un individuo, llevada a cabo normalmente por una asociación profesional o institución académica basada en la competencia demostrada y otros criterios como la experiencia profesional (Mikkelson 2013: 67).

\subsection{Acreditación y estatus socio-profesional}

En otras profesiones consolidadas, como la de abogado o ingeniero, son las propias asociaciones o colegios profesionales las que promueven las certificaciones "voluntarias". Con ellas se persigue ofrecer a los usuarios de los servicios profesionales garantías de que si contratan los servicios de un profesional acreditado, estos serán de calidad. A los profesionales acreditados les garantiza un prestigio y visibilidad profesionales, acceso a bolsas de empleo, asistencia en la movilidad en la Unión Europea, acceso a un seguro de Responsabilidad Civil, formación continua y otras ventajas de índole profesional. ${ }^{20}$ En España no existe actualmente ningún organismo o colegio profesional de intérpretes judiciales que realice dichas funciones.

En España, en el polo opuesto a los perfiles profesionales consolidados y en proceso de continua evolución, se encuentra la interpretación judicial, una actividad que actualmente carece de definición clara, prestigio profesional y reconocimiento social. Por un lado, existen los intérpretes de plantilla (funcionarios o contratados laborales), tanto en el Ministerio de Justicia como en el Ministerio del Interior, que cada vez son menos y que subsisten a duras penas (Ortega Herráez 2011). Por otro lado, en los últimos años, tanto el Ministerio de Justicia como el Ministerio del Interior generalmente se

20. En España existen corporaciones profesionales como COGITI http://www.cogiti.es/ Paginas/Ficha.aspx?IdMenu=A2238BD0-3048-4D9D-AB8C-C91C6FDFD475 
abastecen de "intérpretes" a través de las empresas privadas que han ganado una licitación pública. ${ }^{21}$

El perfil profesional de intérprete judicial que se propone en la actualidad, y en el que se inspira la Directiva cuando se refiere a "intérpretes cualificados e independientes", es el del intérprete judicial como profesional autónomo y que ha obtenido una cualificación específica que le capacita para ejercer en el mercado profesional. Al igual que en una gran mayoría de profesiones liberales, tras obtener un título superior universitario específico que le habilita para el desempeño de su trabajo, el intérprete autónomo busca y obtiene empleo de una variedad de fuentes laborales, tanto en el ámbito judicial o jurídico (casos y mediación civil, notarías), como en el ámbito más generalista de la interpretación de conferencias (congresos, jornadas, cursos) y de la traducción. No es lógico pensar que se pueda subsistir únicamente de una sola fuente de ingresos en el mercado libre, por lo que el intérprete cualificado normalmente trabajará para distintos empleadores, tanto públicos como privados, e incluso diversificará su oferta, ampliando su perfil al de traductor. En estos mismos términos se plantea el perfil de intérprete judicial en Status Quaestionis (Hertog 2008), proyecto financiado por la Dirección General de Justicia de la Comisión Europea que analiza el estado de la interpretación judicial en Europa por medio de cuestionarios respondidos por autoridades judiciales e intérpretes de los Estados Miembros.

\subsection{Acreditación y trastorno del mercado español}

Junto con el desarrollo e implantación de un sistema de acreditación de intérpretes judiciales, el principal obstáculo para la regulación del acceso a la profesión es el fenómeno que Witter-Merithew \& Johnson (2004: 20, apud Mikkelson 2013: 71) denominan trastorno del mercado o market disorder, ${ }^{22}$ del cual España es un buen exponente:

Defined as the current state of the interpreting market that reflects significant instability related to minimum standards for entry into the field and the lack of consistent and reliable professional control over the variables impacting the effective delivery of interpreting services (e.g., introduction into the field, working conditions, job descriptions, role and responsibility, wages).

21. Véase apartado 2.2 y Giambruno (2014: 174).

22. Wallace, en este mismo volumen, relaciona el fenómeno con la ausencia de registros profesionales. 
En el caso español, el problema se ve agravado por la situación de auténtico oligopolio existente en la provisión del servicio, en manos de empresas interesadas en contratar a intérpretes a precios ínfimos con el fin de incrementar sus beneficios. Esto hace que las personas que trabajan como intérpretes no se molesten en obtener una formación, costosa en tiempo y dinero. Sin incentivos como la perspectiva de acceso a una profesión dignamente remunerada y prestigiosa, no habrá intérpretes que deseen formarse y acreditarse, y por tanto que garanticen una mínima calidad de las interpretaciones en los procesos judiciales.

A esta situación hay que añadir la disparidad de denominaciones, títulos e instituciones acreditadoras de intérpretes en España, que aportan confusión al ya escasamente perfilado panorama profesional. Es necesario que los títulos sean unívocos y con descripciones claras acerca de las competencias para las que habilitan. En este sentido, las universidades españolas están realizando un gran esfuerzo por vincularse todavía más a la profesión mediante la colaboración constante con intérpretes en activo, el desarrollo de jornadas y cursos con una clara orientación al mercado profesional, y la incorporación y descripción de las competencias profesionales en sus títulos.

\subsection{Propuesta de acreditación europea: el proyecto QUALITAS}

En el seno de Qualitas: Assessing Legal Interpreting Quality through Testing and Certification (Giambruno 2014), proyecto financiado por el programa Justicia Penal de la Unión Europea para la homogenización del sistema de acreditación de intérpretes judiciales en Europa, catorce expertos de siete países europeos han analizado y diseñado un sistema de acreditación para intérpretes judiciales basándose en experiencias que han funcionado tanto en Europa como en otros países (Estados Unidos, Canadá, Australia) así como en la aplicación de criterios y técnicas psicométricas de desarrollo de pruebas de evaluación.

El proyecto ha realizado un amplio y detallado estudio sobre el sistema de acreditación de intérpretes judiciales, tratando los siguientes puntos:

- Destrezas básicas mínimas y conocimientos legales y profesionales que deberían comprobarse en cualquier sistema de acreditación de intérpretes judiciales

- Principios básicos de diseño de pruebas y psicometría, así como su aplicación en pruebas para intérpretes judiciales con ejemplos

- Criterios para la selección de intérpretes en lenguas de menor difusión

- Aplicación de nuevas tecnologías a la interpretación judicial y policial: Interpretación remota (telefónica o por videoconferencia)

- Organización, administración y gestión de un sistema de acreditación de intérpretes judiciales. 


\subsection{Propuesta de acreditación de intérpretes judiciales en España}

La propuesta de acreditación para España que presentamos a continuación está inspirada en gran medida en el informe de la CCDUTI para el Ministerio de Justicia (Blasco Mayor et al. 2013), y los resultados del proyecto Qualitas (Giambruno 2014).

\subsubsection{Requisitos previos a la acreditación}

Es importante establecer los requisitos previos que deben reunir los candidatos para presentarse al proceso de acreditación. Entre los factores que hay que considerar se incluyen la formación académica, la experiencia laboral acreditada, y algunos criterios de índole personal como pueden ser una edad mínima, la nacionalidad/ciudadanía o el no tener antecedentes penales, entre otros. Estos datos se podrían comprobar en una fase previa al proceso de acreditación por medio de una aplicación informática que excluyese automáticamente a los candidatos que no reuniesen los requisitos establecidos.

\subsubsection{Dominio de los idiomas}

Por la alta capacitación requerida para el ejercicio de la interpretación judicial, creemos que los posibles candidatos a la acreditación deben ser, siempre que sea posible, titulados superiores y demostrar una competencia lingüística de nativo o casi nativo. Así, el nivel C2, según el Marco Común Europeo de Referencia para las Lenguas (MCERL), es el nivel recomendado; sin embargo, es necesario comprobar los niveles reales de los candidatos, incluyendo el manejo de registros, el lenguaje especializado y la terminología técnica, entre otros elementos. Una opción para determinar si se ha alcanzado el nivel requerido es por medio de una "prueba eliminatoria" (screening exam) que puede ser de tipo test, pues es menos costoso que realizar un examen ante un tribunal. Solo las personas que superen este test de nivel lingüístico podrán acceder a la segunda fase del proceso.

\subsubsection{Conocimientos del sistema legal y códigos deontológicos/de buenas prácticas}

En el ámbito de la interpretación judicial, es universalmente reconocido que los procesos de acreditación deben incluir una evaluación de los conocimientos de los sistemas legales y de las normas de comportamiento profesional establecidas. Si un traductor o intérprete no conoce los elementos básicos del sistema en el que va a trabajar (estructura, procesos, derechos, instituciones, 
participantes y demás), la posibilidad de cometer un error al trabajar en un entorno real aumenta exponencialmente.

Lo mismo se puede decir en relación con la deontología. Conocer y comprender los límites del comportamiento ético es imprescindible en este ámbito. La evaluación de estos conocimientos puede incluirse en un ejercicio eliminatorio general para todos los idiomas.

\subsubsection{Elaboración de un instrumento de evaluación de la interpretación}

Se trata de un proceso complejo que debe ser desarrollado por expertos de distintos campos, entre los que se debería incluir a expertos lingüistas, intérpretes experimentados, especialistas en psicometría, juristas y autoridades gubernamentales o representantes de los organismos que tengan funciones de control, supervisión o regulación en el sistema judicial. A continuación se presentan los principales factores que es necesario tener en cuenta en la elaboración de un instrumento de evaluación:

a) Tipo de acreditación y características básicas de las pruebas

Los candidatos deberán acreditarse por separado de cada combinación de lenguas en las que deseen ejercer (español-inglés, español-árabe, españolrumano, etc.). Siguiendo a Van Deemter, Maxwell-Hislop \& Townsley (2014), lo imprescindible en la elaboración de un examen de interpretación es que debe basarse en la ejecución de tareas auténticas, es decir, en la realidad profesional de un intérprete que trabaja en el ámbito judicial (performance-based) y ser evaluado según parámetros preestablecidos, es decir, no condicionados por el número de candidatos o las necesidades del sistema judicial (criterionreferenced). Estos dos conceptos, procedentes de la psicometría, no se pueden modificar si se quiere lograr un examen válido y fiable.

En cuanto a la evaluación, además, es necesario garantizar la fiabilidad entre los evaluadores para garantizar que cada ejercicio reciba un trato similar y que aprobar no dependa del evaluador en particular asignado para evaluar al candidato (inter-rater reliability) ni de los ánimos o situaciones puntuales de un evaluador que reciba decenas de ejercicios para evaluar (intra-rater reliability).

b) Organización administrativa del examen de acreditación en traducción e interpretación judicial

En este apartado se recogen algunos aspectos importantes relacionados con la realización de los procesos de acreditación que no tienen que ver 
específicamente con los ejercicios en sí, pero que son de igual importancia y contribuyen a que el proceso elaborado produzca los resultados deseados:

- Identificar a expertos que puedan formar parte del equipo de elaboración de los exámenes, el personal que va a encargarse de la realización de las sesiones de evaluación, y los evaluadores. Cada grupo debe participar en sesiones de orientación para garantizar una correcta administración de los exámenes.

- Determinar las necesidades logísticas (espacios, equipo informático, acústica, entre otros). El uso de tecnologías digitales en línea reduce en gran medida el coste y procedimiento administrativo de las pruebas, como ha demostrado el programa de evaluación de intérpretes en línea desarrollado por Middlesex University (Braun, Sandrelli \& Townsley 2014: 120).

- Desarrollar materiales para los candidatos con información sobre los requisitos, el proceso de inscripción, el formato del examen, tarifas, plazos, e incluso algunos ejercicios a modo de ejemplo. El desarrollo de esta información será clave para atraer a candidatos cualificados y así evitar el coste elevado de realizar un examen con candidatos que no han logrado todavía los niveles necesarios para alcanzar el éxito esperado en el examen.

Podemos concluir, por tanto, que el proceso de evaluación y acreditación de intérpretes judiciales no es una tarea sencilla. Sin embargo, como se ha demostrado en párrafos anteriores, en los últimos años se ha avanzado mucho en la investigación e implementación de sistemas que tienen en cuenta tanto el objetivo de dicho proceso como las dificultades que entraña. Solo hace falta que los gobiernos de los Estados Miembros se convenzan de que el proceso de evaluación y acreditación de los candidatos a intérprete judicial es esencial para garantizar su competencia.

\section{Formación de operadores judiciales para trabajar con intérpretes}

Por último, pero no por ello menos importante, queremos abordar aquí la cuestión recogida en el artículo 6 de la Directiva 2010/64/UE sobre la formación de personal judicial para trabajar con intérpretes. Concretamente el artículo 6 insta a los Estados Miembros a

...solicitar a los responsables de la formación de los jueces, fiscales y personal judicial que participen en procesos penales el que presten una atención particular a las particularidades de la comunicación con la ayuda de un intérprete, de manera que se garantice una comunicación efectiva y eficaz.

Consideramos que la formación de agentes judiciales tiene una importancia capital si se quiere conseguir la calidad de la interpretación a la que alude la Directiva a lo largo de su articulado. 
Los sistemas de justicia son engranajes complejos en los que intervienen múltiples agentes. En la última década se han multiplicado los procesos penales con elementos extranjeros, "sea personales (víctimas, imputados, testigos, peritos) o materiales (transacciones financieras internacionales, pruebas situadas en otro país, etc.)", según indica Carmona Ruano (2013: IX). En este nuevo escenario judicial, y siguiendo al mismo autor refiriéndose a los jueces,

no podemos permanecer pasivos y permitir que la irrupción de la dimensión internacional en los procesos suponga un obstáculo: por el contrario, hemos de asegurar no solo que los jueces y tribunales de nuestros países puedan afrontar estas circunstancias con normalidad, sino que además seamos capaces de obtener todas las enormes posibilidades que nos ofrecen las nuevas formas de cooperación que se están creando (Carmona Ruano 2013: X).

La normalidad y posibilidades de cooperación a las que alude Carmona Ruano pasan por la indefectible tarea de traslación de información, documentos e intercambios entre jurisdicciones y, por tanto, de unas lenguas a otras. Los jueces, abogados y demás operadores judiciales que trabajan actualmente en el sistema de justicia español rara vez trabajan con intérpretes profesionales, de ahí su absoluto desconocimiento de la actuación y comportamiento de un intérprete profesional, y de cómo realizar su trabajo con la mayor eficacia posible si necesitan la asistencia de un intérprete.

La colaboración entre juristas e intérpretes profesionales es, sin embargo, una reivindicación antigua del colectivo de intérpretes judiciales profesionales españoles, quienes "destacan la imperiosa necesidad de formar adecuadamente a los jueces, fiscales, policías, y en general todos los sujetos llamados a intervenir junto al intérprete" (Vidal Fernández 2007: 224). Es, por tanto, necesaria su formación a través de los cursos que se organizan dentro de sus corporaciones y asociaciones profesionales, y corresponde a las autoridades judiciales trasladar esta función formativa a las mismas. A continuación citaremos las organizaciones judiciales más relevantes en España: Consejo General del Poder Judicial, Red Judicial Española de Cooperación Judicial Internacional (REJUE), Red de Expertos en Derechos de la Unión Europea (REDUE), Red Judicial Europea (RJE), Eurojust. De entre las asociaciones de jueces, magistrados, fiscales y abogados destacan Jueces para la Democracia, Jueces Francisco de Vitoria, Asociación de la Magistratura, Unión Progresista de Fiscales, Asociación de Fiscales, Consejo General de la Abogacía, entre otros.

Igualmente importante es la formación de los Cuerpos y Fuerzas de Seguridad del Estado, pues forman parte del proceso penal durante la fase de instrucción, y su actuación requiere con frecuencia de la asistencia de intérprete. 
Las escuelas más destacadas son la Escuela Nacional de Policía, el Centro de Altos Estudios Policiales y las academias de la Guardia Civil.

No hay que olvidar a los futuros operadores legales que ya pueden recibir formación durante su etapa universitaria a través de las Escuelas de Práctica Jurídica de Colegios de Abogados y Universidades, y también en los Másteres de abogacía, puesto que en su futuro profesional se encontrarán en más de una ocasión con la necesidad de recurrir a un intérprete para entrevistarse con su cliente o con los testigos, o intervenir en un juicio asistido por intérprete.

Por último, se debe incluir en este colectivo a los equipos forenses, que con frecuencia trabajan asistidos por intérpretes en su trabajo cotidiano.

En recientes publicaciones tanto europeas (Corsellis, Clement \& Vanden Bosch 2011; Townsley 2013) como nacionales (Blasco Mayor 2014), orientadas específicamente a la formación de operadores judiciales, se indican las características que debe poseer un intérprete judicial profesional, cómo detectar si el comportamiento del intérprete es profesional, y pautas para trabajar eficazmente con la asistencia de intérprete durante el proceso penal. Mediante un entrenamiento breve pero intensivo, ${ }^{23}$ los operadores judiciales maximizarán el rendimiento de su trabajo al ser asistidos por intérpretes, lo cual redundará en beneficio de todos los implicados, del funcionamiento de la justicia española, y de la salvaguarda de los derechos fundamentales.

\section{Conclusiones}

La construcción de la Unión Europea ha hecho surgir un actor de suma importancia en el concierto internacional al crear un espacio de libertad, seguridad y justicia. Dentro de ese espacio es prioritario garantizar una serie de derechos entre los que se encuentran el derecho a la defensa y el derecho a un juicio justo, los cuales incluyen el derecho a la información de acusados y víctimas y, por ende, el derecho a interpretación y traducción en el caso de las personas que no hablan o no entienden la lengua del procedimiento. La UE ha tomado ya sólidas medidas para garantizar esos derechos, no solo de jure sino también de facto y por ello es importante que los Estados Miembros se rijan por ese mismo espíritu a la hora de transponer las normas europeas a la legislación nacional.

Nos encontramos, pues, en un momento histórico en el que España, como los demás Estados de la UE, tiene la oportunidad de crear un marco legislativo que no solo garantice el derecho a interpretación y traducción en los procesos

23. Véase el artículo de Hale sobre formación de jueces para trabajar con intérpretes en este mismo volumen. 
penales (garantía de jure), sino también que ese derecho sea efectivo mediante un servicio de calidad prestado por profesionales formados y acreditados para tal fin (garantía de facto).

En este sentido, los Estados Miembros deberán crear mecanismos para garantizar la calidad de la interpretación judicial, entre los que cabe destacar un registro profesional, cuyo acceso debería regirse por criterios objetivos, medibles y fiables. El mejor modo de garantizar dichos criterios es desarrollar un sistema de acreditación. En cualquier caso, lo más importante es que el registro sea independiente, que opere a favor de los intereses del sistema de justicia, de los profesionales de la traducción y la interpretación y de la sociedad en general, y que exista un entendimiento claro y consensuado de su cometido y del importante papel que tiene.

Resulta lógico pensar que para que pueda haber intérpretes profesionales cualificados es necesario que exista una oferta formativa que pueda dar respuesta a las demandas reales de nuestra sociedad. Por ello, desde las instituciones responsables de la formación debe existir un compromiso claro con este cometido y articular ofertas que respondan a estas necesidades, tanto en términos de contenidos como de formato.

La creación de un proceso para la acreditación de intérpretes que presten sus servicios en el sistema de justicia es de suma importancia para las partes implicadas directamente en un proceso penal, pero también lo es para la sociedad en general. La inversión inicial en términos de mano de obra y fondos económicos se verá ampliamente compensada por los beneficios para todos, puesto que se establecerá un sistema correctamente configurado y aplicado que agilice los procesos judiciales y aporte calidad y profesionalidad a los mismos.

Es imprescindible implicar y formar a los agentes que intervienen en el proceso penal sobre cómo se trabaja con intérpretes. El sistema judicial es un engranaje del cual forman parte los intérpretes: desde las diligencias previas llevadas a cabo por los cuerpos de seguridad, pasando por los jueces, abogados, secretarios judiciales, equipos forenses; todo el conjunto de operadores que intervienen en el proceso penal debe conocer qué es un intérprete y cómo trabajar con la asistencia de estos profesionales.

Las autoridades europeas han demostrado su voluntad de fomentar la calidad en la interpretación judicial en Europa con la aprobación de directivas sobre los derechos fundamentales de acusados, testigos y víctimas, y financiando costosos proyectos a través de la Dirección General de Justicia de la Comisión Europea. Los gobiernos de los Estados Miembros y las administraciones de justicia deberían demostrar su voluntad de modificar la situación 
mediante los mecanismos legales que tienen a su disposición, ya que la defensa de los derechos fundamentales recae finalmente en los propios Estados.

\section{Bibliografía}

Arangüena Fanego, Coral. (2007) "Conclusiones." En: Arangüena Fanego, Coral (ed.) 2007. Garantías procesales en los procesos penales en la Unión Europea. Valladolid: Lex Nova, pp. 389-394.

BAXTER, Robert Neal. (2014) "Undergraduate Interpreter Training in the Spanish State: An Analytical Comparison.” Sendebar 25, pp. 219-246.

Blasco Mayor, María Jesús; Maribel del Pozo Triviño; Cynthia Giambruno; Anne Martin; Emilio Ortega Arjonilla; Nadia Rodríguez Ortega \& Carmen Valero Garcés. (2013). Informe sobre la transposición de la Directiva 2010/64/ UE del Parlamento Europeo y del Consejo relativa al derecho a interpretación y traducción en los procesos penales. Versión electrónica: https://www.academia. edu/8755014/Informe_sobre_la_transposición_de_la_Directiva_2010_64_ UE_del_Parlamento_Europeo_y_del_Consejo_relativa_al_derecho_a_ interpretación_y_traducción_en_los_procesos_penales

Blasco MAYOR, María Jesús. (2013). "Quality of interpreting in criminal proceedings in Spain under European Directive 2010/64/EU". Cuadernos de ALDEEU 25, pp. 165-190.

Blasco MAYOR, María Jesús. (2014) "La asistencia de intérprete en el procedimiento penal. Especial referencia a su papel en la vista oral." En: Cuerda, María Luisa (dir.) \& Antonio Fernández Hernández (ed.) 2014. Vistas penales. Casos resueltos y guías de actuación en sala. Valencia: Tirant Lo Blanch, pp. 275-293.

Braun, Sabine; Annalisa Sandrelli \& Brooke Townsley. (2014) "Technological support for testing." En: Giambruno, Cynthia (ed.) Assessing Legal Interpreter Quality through Testing and Certification: The Qualitas Project. Alicante: Universidad de Alicante, pp. 109-139.

Carmona Ruano, Miguel. (2013) "Prólogo." En: Carmona Ruano, Miguel; Ignacio U. González Vega \& Víctor Moreno Catena (dirs.) \& Amaya Arnáiz Serrano (ed.) 2013. Cooperación Judicial Penal en Europa. Madrid: Dykinson, pp. IX-XI.

Corsellis, Ann, Jan Cambridge; Nicky Glegg \& Sarah Robson. (2007) "Establishment, maintenance and development of a national register". En: Wadensjö, Cecilia; Birgitta Englund Dimitrova \& Anna-Lena Nilsson (eds.) 2007. The Critical Link 4. Professionalisation of interpreting in the community. Amsterdam: John Benjamins, pp. 139-150.

CORSEllis, Ann; Amanda Clement \& Yolanda Vanden Bosch. (2011) "The training of members of the legal services working through legal interpreters and 
translators." En: Townsley, Brooke (ed.) 2011. Building Mutual Trust: A framework project for implementing EU common standards in legal interpreting and translation. Londres: Middlesex University, pp. 278-316.

DE LUNA JIMÉNEZ DE PARGA, Pilar. 2009. Informe sobre la calidad de la interpretación en los Juzgados de lo Penal de Madrid. Versión electrónica: http://www.elgas conjurado.com/2010/02/15/informe-de-la-magistrada-pilar-de-luna-jimenezde-parga/

Del Pozo Triviño, Maribel. (2013): "La interpretación en el contexto español actual. Nuevos retos para el futuro". En: Alonso Aragúas, Icíar; Jesús Baigorri Jalón \& Helen Campbell (eds.) 2013. Translating the Law. Theoretical and Methodological Issues. / Traducir el Derecho. Cuestiones teóricas y metodológicas. Granada: Comares, pp. 57-65.

Del Pozo Triviño, Maribel \& Anabel Borja Albi. (2014) "Court translation and interpretation in Spain. Towards a better future!” En: Bathia, Vijay K; Giuliana Garzone; Rita Salvi; Girolamo Tessuto \& Christopher Williams (eds.) 2014. Language and Law in Academic and Professional Settings: Analyses and Applications. Aprilia: Novalogos, pp: 219-232.

DueÑas GonZÁlez, Roseann, Victoria F. Vásquez \& Holly Mikkelson. (1991) Fundamentals of Court Interpreting. Theory, Policy and Practice. Durham: Carolina Academic Press.

Giambruno, Cynthia. (ed.) (2014) Assessing Legal Interpreter Quality Through Testing and Certification: The Qualitas Project. Alicante: Universidad de Alicante.

GILE, Daniel. (2009) Basic Concepts and Models for Interpreter and Translator Training. Amsterdam: John Benjamins.

HANDI, Elhassane Benhaddou. (2012) "La traducción y la interpretación en el Ministerio del Interior." En: Alonso Araguás, Icíar; Jesús Baigorri Jalón \& Helen Campbell (eds.) 2012. Ensayos sobre la traducción jurídica e institucional. Granada: Comares, pp. 91-102.

Hertog, Erik. (ed.) (2001) Grotius project 98/GR/131. Aequitas. Equal Access to Justice across Language and Culture in the EU. Amberes: Lessius Hogeschool.

HertoG, Erik \& Jan Van Gucht. (eds.) (2008) Status Quaestionis: Questionnaire on the Provision of Legal Interpreting and Translation in the EU. AGIS project JLS/2006AGIS/052. Amberes: Intersentia.

MiKKELSON, Holly. (1996) "Community interpreting: an emerging profession." Interpreting 1:1, pp. 125-129.

MikKelsON, Holly. (2013) "Universities and Interpreter Certification". The International Journal for Translation and Interpreting Research 5:1, pp. 66-78.

MikKeLSON, Holly. (2014) "Evolution of Public Service Interpreter Training in the U.S." FITISPos International Journal 1:1, pp. 9-22.

Ortega Herráez, Juan Miguel \& Ana Foulquié Rubio. (2008) "Interpreting in police settings in Spain". En: Valero Garcés, Carmen \& Anne Martin (eds.) 
Crossing Borders in Community Interpreting. Amsterdam: John Benjamins, pp. 123-146.

Ortega HerRÁEZ, Juan Miguel. (2011) Interpretar para la justicia. Granada: Comares.

PeÑarroja FA, Josep. (2004) "Historia de los intérpretes jurados." La linterna del traductor 9. Versión electrónica: http://traduccion.rediris.es/6articulos_a.htm

TOWNSLEY, Brooke (2013) Building Mutual Trust 2. Versión electrónica: http:// www.buildingmutualtrust.eu/

VAN DEEMTER, Roelof, Hilary Maxwell-Hyslop \& Brooke Townsley. (2014) "Principles of testing." En: Giambruno, Cynthia (ed.) 2014. Assessing Legal Interpreter Quality through Testing and Certification: The Qualitas Project. Alicante: Universidad de Alicante, pp. 27-39.

VARIOS AUTORES (RITAP - Red de Intérpretes y Traductores para la Administración Pública). (2011). Libro blanco de la traducción y la interpretación institucional. Madrid: Ministerio de Asuntos Exteriores y Cooperación. Versión electrónica: http://ec.europa.eu/spain/pdf/libro_blanco_traduccion_es.pdf

VIDAL FERNÁNDEZ, Begoña. (2007) "Derecho a una interpretación y traducción fidedigna y de calidad. Artículos 8 y 9 de la Propuesta de Decisión Marco sobre las garantías procesales de los inculpados en procesos penales en la Unión Europea." En: Arangüena Fanego, Coral (ed.) 2007. Garantías procesales en los procesos penales en la Unión Europea. Valladolid: Lex Nova, pp. 214-231.

VIGIER MORENO, Francisco Javier. (2010) El nombramiento de traductores-intérpretes jurados de inglés mediante acreditación académica: descripción de la formación específica y del grado de satisfacción de los egresados. Granada: Universidad de Granada. Tesis doctoral inédita.

Witter-Merithew, Anna \& Leilani Johnson. (2004) "Market Disorder Within the Field of Sign Language Interpreting: Professionalization Implications". Journal of Interpretation 14, pp. 19-55.

\section{BIONOTA / BIONOTE}

María Jesús Blasco Mayor es intérprete y traductora titulada por la Universidad de Granada. Es profesora de interpretación en el Grado y en el Máster de Investigación en Traducción e Interpretación de la Universitat Jaume I. La investigación realizada para su tesis doctoral versó sobre bilingüismo y el proceso cognitivo de la comprensión en la interpretación. Ha participado en numerosos proyectos de investigación sobre calidad de la interpretación y sobre interpretación y traducción judicial. Ha publicado en prestigiosas revistas académicas, así como editado varios volúmenes sobre interpretación 
y bilingüismo, cognición, interpretación judicial y policial, y el uso de nuevas tecnologías.

María Jesús Blasco Mayor is an interpreter and translator with a degree from the University of Granada who has been teaching interpreting since 1997 at Universitat Jaume I (Spain). Her PhD research centered on the comprehension component of conference interpreter training from a cognitive standpoint. She has participated in several research projects on interpreting quality and legal translation and interpreting. She has published in leading journals, authored and edited volumes on her research interests, which include cognitive processes in interpreting training, interpreting quality, court and police interpreting in Spain, and the use of new technologies in interpreting.

Maribel del Pozo Triviño es Doctora en Traducción e Interpretación y Traductora e Intérprete Jurada inglés-español. Ha desarrollado una intensa carrera profesional como traductora e intérprete y actualmente es profesora en la Universidad de Vigo. Tiene numerosas publicaciones relacionadas con la traducción jurídica y la traducción e interpretación en los servicios públicos y participa en varios proyectos de investigación relacionados con este ámbito. Es coordinadora del proyecto europeo Speak Out for Support (SOS-VICS), centrado en la formación de intérpretes para trabajar con víctimas de violencia de género. Forma parte del grupo de investigación GENTT, es miembro de AIETI, ATIJC, APTIJ, AGPTI y de la Red Comunica.

Maribel del Pozo Triviño has a PhD in Translation and Interpretation and she is a sworn English-Spanish translator-interpreter. She has worked as a translator and interpreter for many years and is currently working as a lecturer at the University of Vigo. She has published extensively on different aspects of legal translation and public services translation \& interpretation and is currently involved in several research projects on the mentioned subjects. She is the coordinator of the EU project Speak Out for Support (SOS-VICS), focused on training interpreters to work with victims of gender violence. She is a member of GENTT research group, AIETI, APTIJ, ATIJC, AGPTI and the Comunica Network. 